\title{
A Previously Unreported Arterial Variant of the Suboccipital Region Based on Cadaveric Dissection
}

\author{
Christian Fisahn ${ }^{1,2}$ Brittni Burgess ${ }^{2}$ Joe Iwanaga ${ }^{2}$ Fernando Alonso $^{1}$ Jens R. Chapman ${ }^{1}$ \\ Rod J. Oskouian ${ }^{1}$ R. Shane Tubbs ${ }^{2}$
}

${ }^{1}$ Swedish Medical Center, Swedish Neuroscience Institute, Seattle, Washington, United States

2 Seattle Science Foundation, Seattle, Washington, United States

\begin{abstract}
Address for correspondence Christian Fisahn, MD, Swedish Medical Center, Swedish Neuroscience Institute, 550 17th Avenue, Seattle, WA 98122, United States (e-mail: christian.fisahn@gmail.com).
\end{abstract}

J Neurol Surg Rep 2017;78:e37-e39.

\author{
Abstract \\ Keywords \\ - anatomy \\ - anomaly \\ - carotid artery \\ - C1 \\ - suboccipital triangle
}

Introduction Several arterial variants have been reported to occur around the posterior arch of the atlas. Understanding the various anomalies and diagnosing them preoperatively can dramatically reduce the risk of surgical insult during neurosurgical procedures. Herein we report a case of an arterial variant found just below the posterior arch of $\mathrm{C} 1$.

Case Report During the routine dissection of the suboccipital region via a posterior approach, an unusual bulge was identified just inferior to the inferior capitis oblique muscle. With further dissection, the structure was identified as a tortuous internal carotid artery.

Conclusion Arterial variants around the posterior arch of $\mathrm{C} 1$ are surgically significant and can result in catastrophic injuries if unappreciated. Most of these variants will be related to the vertebral artery. To our knowledge, an arterial variant of the internal carotid artery in this location, as reported herein, has not been previously reported.

\section{Introduction}

The atlas is surrounded by several arterial anomalies, and it has a unique shape involving a complex relationship with the vertebral artery (VA). ${ }^{1}$ The complexity of its architecture poses potential of a high complication risk for surgeries occurring in the atlas region. Having a complete understanding of the various anomalies preoperatively can dramatically reduce the risk of surgical insult to them during neurosurgical procedures. Some of the arterial anomalies reported to occur around the atlas include posterior-inferior cerebellar artery (PICA) of an extradural origin, persistent proatlantal and hypoglossal arteries, and VAs passing through arcuate foramina. ${ }^{2-4}$ Herein we present a rare case of an arterial variant found just inferior to the suboccipital triangle.

\section{Case Report}

During the routine dissection of the right suboccipital region in a adult male cadaver as part of a skull base course, a large 1-cm-diameter bulge was noted just inferior to the suboccipital triangle (i.e., just inferior to the inferior capitis oblique muscle) (-Fig. 1). This vascular structure was appreciated after the semispinalis capitis muscle had been reflected inferiorly. This muscular triangle was slightly compressed inferiorly. Initially, the variant vessel was thought to be a malpositioned and enlarged VA. However, the ipsilateral VA was found to be in a normal location within the suboccipital triangle and passed over the posterior arch of the atlas as it normally does. It was of a normal caliber. No other neurovascular or muscular variants were identified on the left or received

October 27, 2016

accepted after revision

February 10, 2017
DOI http://dx.doi.org/ 10.1055/s-0037-1600136. ISSN 2193-6366. (c) 2017 Georg Thieme Verlag KG Stuttgart · New York
License terms

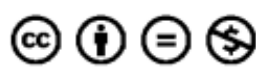




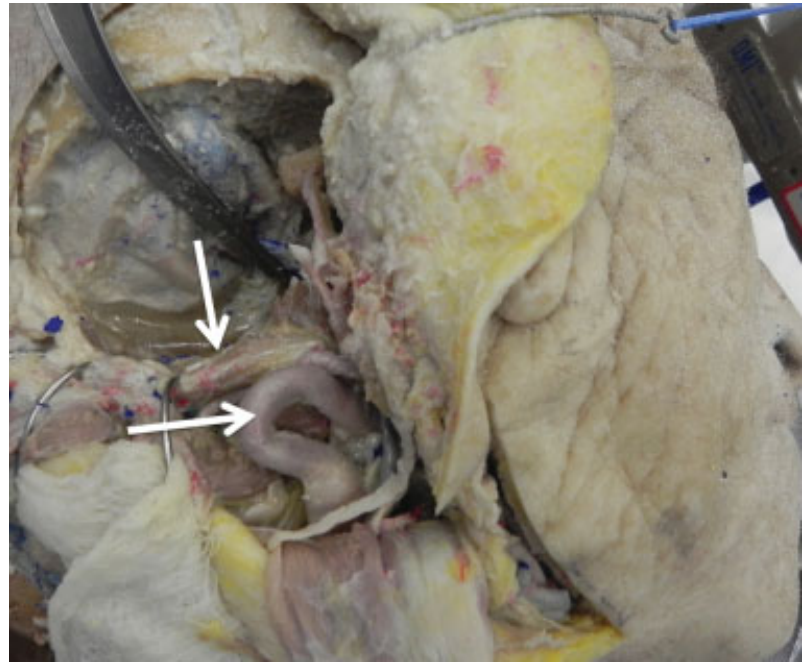

Fig. 1 Right lateral skull base dissection. The large aberrant loop of the internal carotid artery is shown (horizontal arrow) bulging into the suboccipital region inferior to the inferior capitis oblique muscle (vertical arrow).

right sides of the specimen. With further dissection, the vascular structure was shown to be the distal part of the internal carotid artery. This vessel course more posteriorly than usual came into the suboccipital region as a large loop and then traveled superiorly into the carotid canal-the latter being in normal fashion.

\section{Discussion}

Arterial variations involving vessels around the atlas are not uncommon. For example, extracranial-extradural variants of the PICA have been reported from 5 to $20 \%$ during lateral suboccipital, far-lateral, or upper cervical approaches. ${ }^{1,5,6}$ The course of this vascular variant is of surgical importance as it can be confused with V3 branches (muscular, meningeal, posterior spinal). Common persistent carotid-vertebrobasilar anastomoses that might be observed in the suboccipital region include persistent hypoglossal and proatlantal arteries. The hypoglossal artery arises from the cervical internal carotid artery at the $\mathrm{C} 1-\mathrm{C} 3$ level, extends through the hypoglossal canal and joins with the basilar artery close to the pontomedullary junction, and has a prevalence of 0.02 to $0.1 \%{ }^{5}$ The proatlantal artery, thought to be manifested as the horizontal segment of the adult VA, can arise from the internal or external carotid arteries and joins distally with the vertebrobasilar system. , $^{3,7}$

Our case is unique in that the cervical internal carotid artery was found to enter the suboccipital region as a large looped vessel before continuing along its normal course to enter the carotid canal. Damage to the vessel at this point could have devastating outcomes. The presence and route of such arterial variants can be best confirmed with a three-dimensional computed tomography angiography (3D-CTA) to avoid any injury to them during common neurosurgical procedures such as midline approaches to the posterior cranial fossa, $\mathrm{C} 1-\mathrm{C} 2$ screw fixation, and $\mathrm{C} 1 / 2$

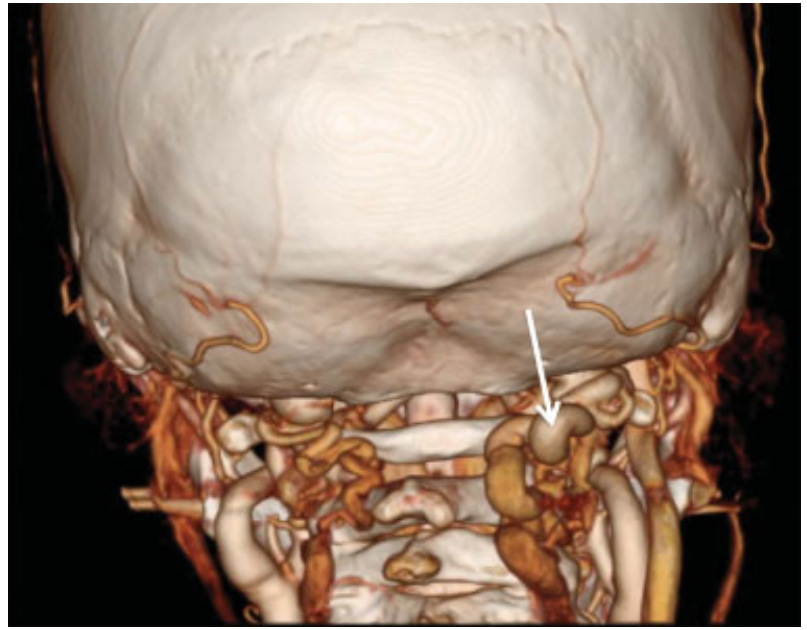

Fig. 2 CTA of a patient found to have a similar bulge of artery (arrow) into the suboccipital region most likely representing the finding as seen in our cadaveric specimen.

puncture, a procedure commonly performed to collect cerebral spinal fluid. ${ }^{6,8}$ Interestingly, after identifying our case, a review of our hospital's database identified an adult patient with a similar finding in the suboccipital region that appears to be consistent with our cadaveric case (-Fig. 2). This case was found incidentally for evaluation of headache. Although cases of significant tortuosity of the cervical part of the internal carotid artery have been reported, ${ }^{9}$ known to our knowledge, they have been so deviated as to enter the suboccipital region inferior to the posterior arch of $\mathrm{C} 1$.

\section{Conclusion}

Atlas arterial variants are surgically significant and may influence approaches for several upper cervical spine pathologies. Arterial anomalies can be identified preoperatively utilizing 3D-CTA to minimize risk of complications during neurosurgical procedures. When not performed, the surgeon should be aware of the possibility of the rare variant identified in the current case to minimize complications and avoid morbidity.

\section{Disclosure}

The authors report no conflict of interest concerning the materials or methods used in this study or the findings specified in this paper.

\section{Informed Consent}

Written informed consent was obtained from the patient for publication of this case report and accompanying images. A copy of the written consent form is available for review by the Editor-in-Chief of this journal on request. 


\section{References}

1 Fine AD, Cardoso A, Rhoton AL Jr. Microsurgical anatomy of the extracranial-extradural origin of the posterior inferior cerebellar artery. J Neurosurg 1999;91(04):645-652

2 O’Donnell CM, Child ZA, Nguyen Q, Anderson PA, Lee MJ. Vertebral artery anomalies at the craniovertebral junction in the US population. Spine 2014;39(18):E1053-E1057

3 Montechiari M, Iadanza A, Falini A, Politi LS. Monolateral type I proatlantal artery with bilateral absence of vertebral arteries: description of a case and review of the literature. Surg Radiol Anat 2013;35(09):863-865

4 Yamazaki M, Okawa A, Hashimoto M, Aiba A, Someya Y, Koda M. Abnormal course of the vertebral artery at the craniovertebral junction in patients with Down syndrome visualized by threedimensional CT angiography. Neuroradiology 2008;50(06):485-490

5 Kirkland JD, Dahlin BC, O'Brien WT. The transclival artery: a variant persistent carotid-basilar arterial anastomosis not previously reported. J Neurointerv Surg 2016 neurintsurg2016-012464.rep

6 Wakao N, Takeuchi M, Nishimura M, et al. Vertebral artery variations and osseous anomaly at the C1-2 level diagnosed by 3D CT angiography in normal subjects. Neuroradiology 2014; 56(10):843-849

7 Cohen JE, Grigoriadis S, Itshayek E. Type II proatlantal artery (occipital subtype) with bilateral absence of the vertebral arteries. Clin Anat 2011;24(08):950-952

8 Lasjaunias P, Vallee B, Person H, Ter Brugge K, Chiu M. The lateral spinal artery of the upper cervical spinal cord. Anatomy, normal variations, and angiographic aspects. J Neurosurg 1985;63(02): 235-241

9 Peeling L, Fiorella D. Balloon-assisted guide catheter positioning to overcome extreme cervical carotid tortuosity: technique and case experience. J Neurointerv Surg 2014; 6(02):129-133 